\title{
XVIII. Nanotechnology in automobiles
}

Modern civilizations rely heavily upon motorized vehicles to transport both people and goods. The transport of people from one point to the other all over the world is a major part of the global economy and of the modern lifestyle. The rapid growth in the number of automobiles, first observed in developed countries, and now in developing countries reflects both the desire and the necessity for convenient and flexible on-demand transportation.

In the automotive area, as in others, nanotechnology is applied to produce materials with novel structural and functional properties tailored to a particular need. These materials are used to improve safety and reliability, to reduce weight without sacrificing structural integrity, to reduce energy consumption, to reduce cost, etc. Improvements in comfort and driving pleasure are also important aspects to which drivers and passengers are responsive.

Some of the areas in which nanotechnology offers the possibility of improvement are shown in figure 132. Safety, environmental protection and comfort are the main areas where nanotechnology can have a large impact.

Safety is of course the most important point. Each year, about 1.3 million people in the world die in car or truck accidents. About 50 million are injured. This figure is similar to the population of South Korea.

It is also important to note that road transportation relies heavily on fuels to propel our vehicles. Half of the oil extracted in the world is used to propel vehicles and more than $95 \%$ of the fuel used in road vehicles is derived from oil. Burning oil, in the form of gasoline or diesel, leads to the emission of carbon dioxide $\left(\mathrm{CO}_{2}\right)$, particles, unburned hydrocarbons and carbon monoxide (CO). These emissions can have serious negative effect on the environment and human health. Decreasing fuel consumption by filtering or treating the gases emitted from the exhaust pipe can reduce this pollution. Nanostructured filters and nanocatalysts can help in this respect.

Nanotechnology is already important in the automotive industry. Examples in Figure 133 identify current applications of nanotechnology in many parts of a car.

\section{Bodywork}

Fuel consumption and safety can be improved using nanomaterials. For that it is necessary to reduce the weight of the vehicle while maintaining or improving the performance of the affected components. 


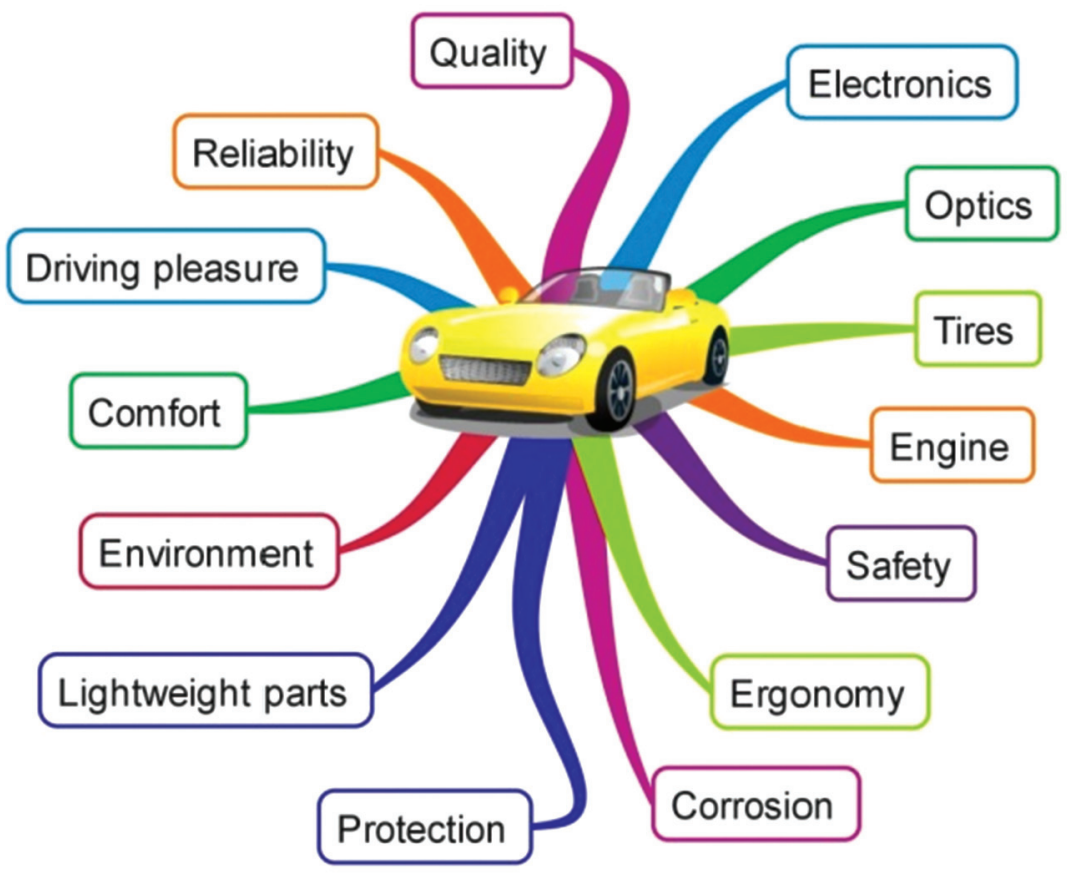

Figure 132. Different areas of the automotive domain that nanotechnology can improve

High-strength flexible parts could be used as crash-absorbers reducing the impact of a collision. Nanotechnology is already employed to manufacture high-strength steel using 5-10 $\mathrm{nm}$ embedded metallic carbon nitride particles. This steel is fatigue-resistant and permits the manufacturing of lighter parts. The development of other fatigue-free nanosteels is envisaged in the near future.

Another important issue is corrosion protection. Galvanic coating processes using chromium VI are now forbidden because they are dangerous for human health and the environment. Chromium III protection processes are far less efficient. Using a three-layer protection: zinc, chrome III and a surface coating of silica nanoparticles of, provides outstanding protection against corrosion and, in addition, provides self-healing properties against small damages to the surface.

In the future, part of the body of the car will be covered with enhanced self-healing materials based on nanotechnologies. Micro-encapsulated healing molecules and a polymerization catalyst put into a common matrix, but separated from each other will be used. Any damage which cracks the surface will break the microcapsules. The healing agent they contain 


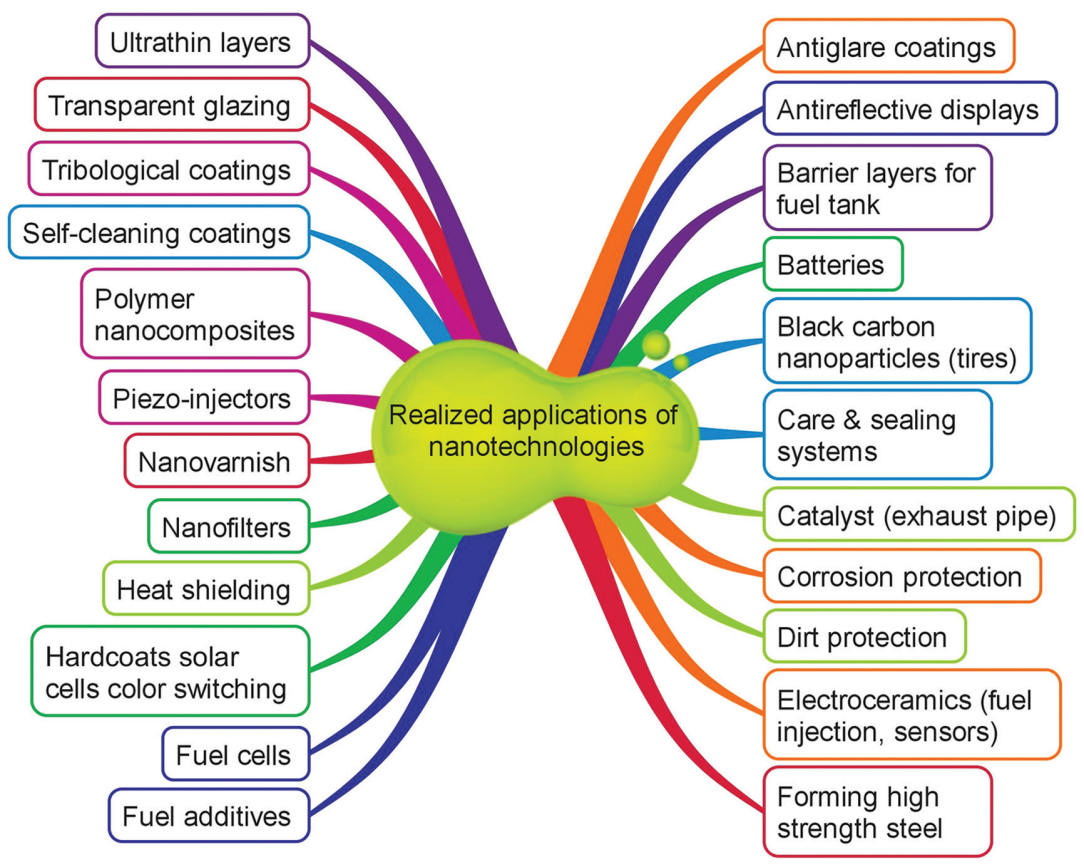

Figure 133. Examples of current applications of nanotechnology in the automotive domain.

will be released and fill the crack. This technique is exemplified by the work of White and al. schematically illustrated in figure 134 .

In the past soldering or bolting was extensively used to build a car. Gluing, increasingly being used in the automotive industry, provides the advantages of low cost and high-processing speeds. Gluing often requires warming the two components to temperatures of the order of $180^{\circ} \mathrm{C}$. This can induce adverse thermal stresses in the components. Using glues containing nanoferrites, it is possible to trigger the gluing process by microwave radiation at wave lengths in which the interaction occurs with the glue only. This requires less energy than conventional gluing and is faster. Consequently the thermal stress to the components is substantially decreased. This technique is called "gluing on demand". Another useful technology for repair is to use a glue which has the ability to detach (unglue) on command. Biologically inspired materials based on the Gecko effect discussed in chapter 5 can provide the "stick and detach on demand" capability. These materials are nanostructured.

The body of a car represents a large surface exposed to external conditions. Using nanotechnology, it is possible to improve several properties or characteristics of the body. By functionalizing the surface with specific 


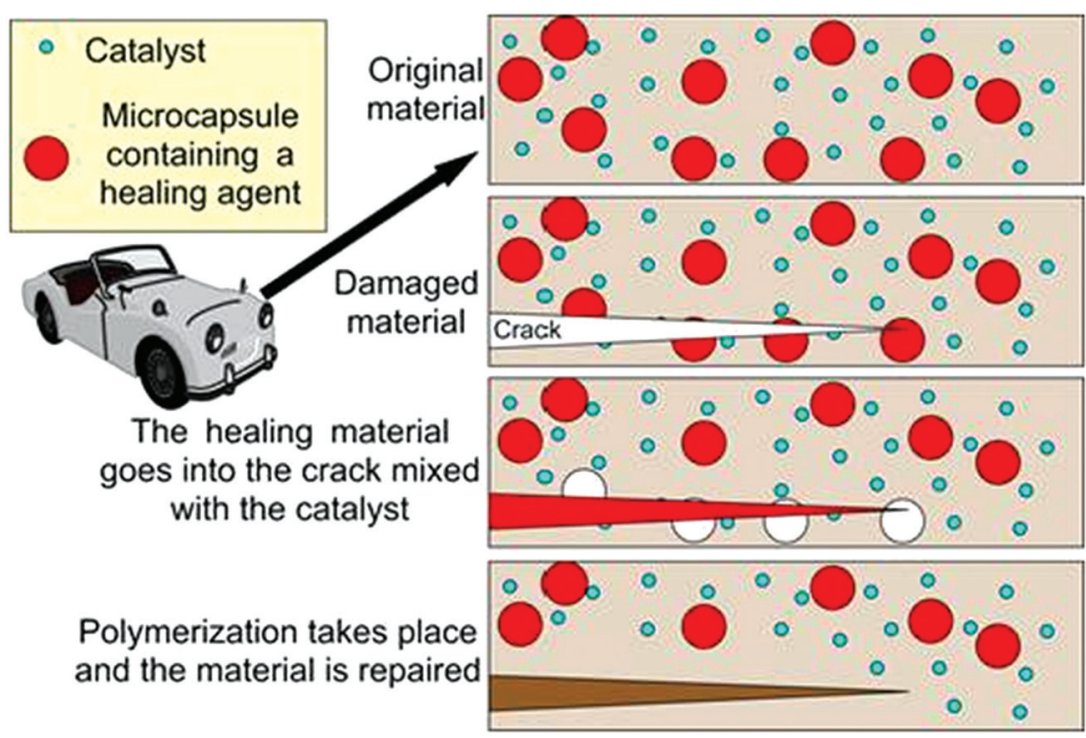

Figure 134. The use of a microencapsulated healing agent embedded in a structural composite matrix containing a catalyst capable of polymerizing that healing agent makes it possible to repair a small crack occurring in the body of a car. During crack formation, the microcapsules are ruptured, releasing healing agent into the crack by capillary action. In contact with the catalyst, the healing agent polymerizes and the crack is repaired.

molecules it is possible to adjust the wetting properties or to protect the surface against external stresses. Silica nanoparticles with a diameter ranging from 7 to $40 \mathrm{~nm}$ used in car paints can make the paint very hard as the paint dries because of a crosslinking mechanism between the nanoparticles. It is 3 times more resistant to scratches. The color of the paint becomes more brilliant. A nanovarnish containing ceramic nanoparticles deposited on the paint can further increase the scratch resistance and maintain the brilliance for a longer time.

Cars have a large area of glass and plastics, windows, mirrors and headlights. Nanostructuration of their surfaces at the nanoscale provides the ability to create surfaces with a gradient in the refractive index. This technology produces optical surfaces with reduced reflection and improved transmission. A good quality reflecting coating is obtained with a nanometer-thickness aluminum oxide layer. Lenses for headlight covers are usually made of polycarbonate which is an extremely hard material and is lightweight. However, it has poor scratch resistance. Coating polycarbonate with paints containing aluminum oxide nanoparticles produces a high scratch resistance, while retaining high optical transparency. It is also possible to have a hydrophobic and oleophobic treatments, based on 
nanometer-thickness layers, to make it easier to clean dirt, fingerprints, sweat deposited on the surface. Self-cleaning surfaces are interesting for glazing, wing mirrors, headlights, alloy wheels, bumpers, etc.

Nanotechnology can be used to produce electrochromic windows whose optical properties change with an applied voltage. This is useful to maintain interior temperatures or for reducing glare from the rear-view mirror of a car at night. This increases safety and comfort of the vehicle for the driver.

It is difficult to keep a car clean. The body and interior have to be cleaned regularly. Anti-dirt products based on nanoparticles are now available. Water and fat repellent nanoparticles deposited on metal, fabrics, glass or plastics can prevent dirt, water or fats from sticking to their surfaces.

\section{Interior of the car}

Using nanomaterials or nanostructured materials mimicking the Gecko effect, it is possible to manufacture fabrics which are dirt-resistant because of their hydrophobic and oleophobic surfaces. These surfaces are self-cleaning, a property interesting for car seats. Photocatalytic materials, such as $\mathrm{TiO}_{2}$ nanoparticles, can decompose dirt. Nanotextile materials have an important future in cars by notably improving the quality of conventional fabrics made of natural or synthetic fibers. Nanocapsules containing a fragrance can be embedded in the fabrics of seats. The fragrance is progressively liberated at each mechanical stress.

Nanofiltration can be used in two applications. The first is control allergenic particles, pollutants or odors. For that nanofilters made of nanofibers are more efficient than conventional filters. Nanofilters are also useful to reduce pollutants emitted by the exhaust pipe. Nanocatalysts, providing much larger areas in contact with the chemical products to be treated, transform the pollutant emitted in the exhaust pipe into harmless molecules.

\section{Chassis and tires}

Tires must reconcile opposite properties: they need to have a good road grip, but a low-rolling resistance; they should be resistant against abrasion, but prevent the car from sliding. Tires based on natural rubber have architectures and additives that give them the good properties currently manifested. They are much different than the tires made in the past. Additives 
such as carbon black, silica, or organosilane nanoparticles are essential to produce tires of excellent quality. The sizes and geometries of the nanoparticles used have a great influence on the final properties of the tire. For example, the introduction of carbon black nanoparticles has improved abrasion resistance and increased the lifetime of a tire by a factor of five. Typically, the thickness of a tire decreases about $5 \mathrm{~mm}$ after $50,000 \mathrm{~km}$ of driving. In the nineties, silica nanoparticles were introduced into the tire manufacturing process. They have significantly increased wear resistance.

The viscosity of a dilatant material increases with the rate of the shear strain. This kind of material, called a shear thickening fluid, is an example of a fluid in which conventional laws of hydrodynamics fail to apply. For that reason, they belong to a wider class of fluids called non-Newtonian fluids. Yoghurt is a common example of non-Newtonian fluid: its viscosity depends on the way you have mixed it with a spoon. A dilatant material can be a colloid containing nanoparticles, which are organized in ordering layers thanks to the Van der Waals interactions. If the shear forces happen to be more important than the Van der Waals forces, flocculation occurs and the fluid behaves more like a solid and is highly viscous. The viscosity of a dilatant fluid changes because the shear stress induces a phase transition from an ordered state to a disordered state so the material looks more like a solid. Dilatant fluids made of a dispersion of nanoparticles can have several applications in the automotive area. They can be used as damping systems or for shock absorption. They can be used in all-wheel-drive coupling, in pedestrian protection, etc.

\section{Power train}

Nanotechnology is also present in many parts of the power train. The aim is to produce more energy-efficient, more environmental-friendly, safer and more comfortable vehicles.

Nanocrystalline piezo-injectors can be used in diesel vehicles, allowing control of the injection valve with an accuracy in the nanometer range. Friction of the pistons of cylinders of the engine waste energy and reducing this friction is an important goal. A nanocrystalline material is able to do that providing the additional advantage of decreasing abrasion and increasing the lifetime.

More generally, nanocoatings are of interest because they are wear-resistant and lead to hardened surfaces. They are particularly useful for highly stressed components such as bearings, cylinders or valves of the powertrain. 
Removing waste heat of the vehicle powertrain is still an important issue because of a regular increase of thermal power density. Increasing the heat exchange surface of a vehicle radiator is not desirable because it increases the weight and the size of the device. Therefore, it is necessary to look at new coolants and thermal coupling materials such as nanofluids. A nanofluid is a liquid containing dispersed and suspended particles with sizes in the nanometer range. It has been shown that an inclusion of these nanoparticles increases the thermal conductivity. Studies are also underway to improve thermal and rheological properties of lubricants by adding nanoparticles.

\section{Electronics}

Electronics has become of ever increasing importance in our vehicles. Electronics accounted for about $7 \%$ of a car's value in 1970, $20 \%$ in 2005 and $40 \%$ in 2010. Most of electronics used today are based on microprocessors etched with dimensions smaller than $100 \mathrm{~nm}$. Electronic sensors are important to maintain a proper functioning of the internal combustion engine. Electronic components made of nanolayers of semiconducting materials are used for engine control, but also in other parts of the car such as airbags, anti-lock brakes and stability control of the vehicle.

European Community legislation requires the electronic industries to eliminate lead from solder, which was traditionally an alloy of tin and lead $(\mathrm{Sn}-\mathrm{Pb})$. New alloys have been developed. One new alloy is an eutectic made of tin, silver and copper. It requires temperatures around $235^{\circ} \mathrm{C}-245^{\circ} \mathrm{C}$, that is about $35^{\circ} \mathrm{C}$ higher than for $\mathrm{Sn}-\mathrm{Pb}\left(210^{\circ} \mathrm{C}\right)$. The higher temperature of operation induces more stress on the electronic components being assembled. Therefore, efforts are being made to reduce the operating temperature. A promising research track appears to be the addition of nanoparticles. The melting points of metals decrease as the sizes of the nanoparticles decreases. Drops of tens of degrees can be obtained using nanoparticles with sizes below $10 \mathrm{~nm}$.

\section{Batteries and supercapacitors}

Batteries can store energy. The development of plug-in hybrid vehicles and electric vehicles requires having reliable batteries with high energy densities per unit of mass. Improvement of batteries is obtained using 
Capacitor discharged

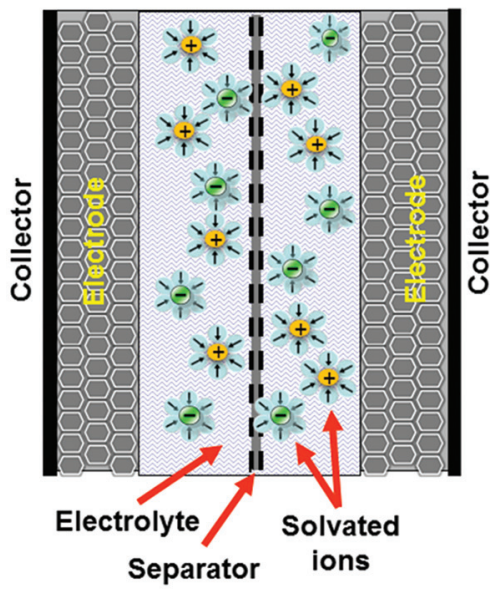

Random distribution of ions

\section{Capacitor charged}

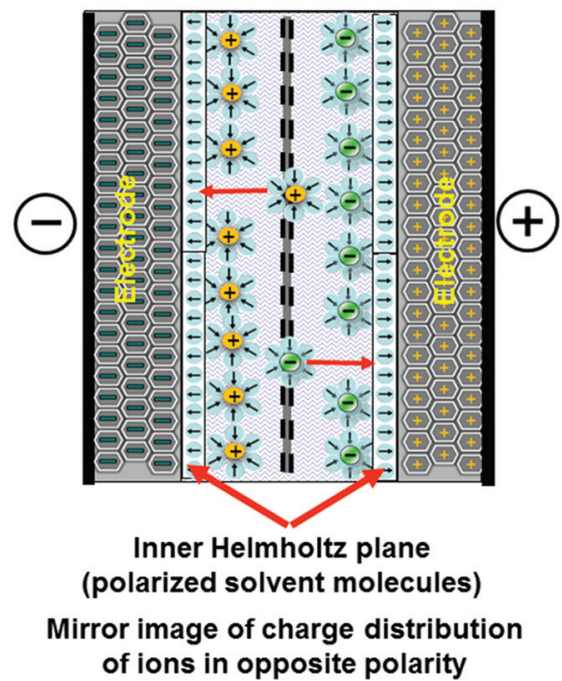

Figure 135. Distribution of ions in a discharged/charged supercapacitor. Image from Wikimedia Commons (http://commons.wikimedia.org). Author Elcap.

nanocomposite plastic to produce solid polymer electrolytes, but also by nanostructuring the electrodes. Typically, a car needs about $15 \mathrm{kWh}$ to travel $100 \mathrm{~km}$ and lithium-ion batteries used in electric vehicles have an energy density of about $120 \mathrm{Wh} / \mathrm{kg}$. The smaller energy density of Li-Ion batteries dedicated to vehicles compared to those that are manufactured for portable devices such as cell phones, laptops, etc. which have around 200 $\mathrm{Wh} / \mathrm{kg}$, is for safety reasons. Indeed, in the case of overloading, the Li-Ion technology used for portable devices can often lead to accidents or damage to the battery. Considering the large amount of energy stored in the battery of a car, similar accidents would have serious consequences.

Supercapacitors can also be used in electric and plug-in hybrid vehicles. They store a small amount of energy but are able to deliver a large amount of power. For that reason, they are interesting to provide fast acceleration to a vehicle. Compared to batteries, they can make a very large number of charge-discharge cycles (more than 100,000). The principle of a supercapacitor is shown in figure 135 .

Nanomaterials and nanostructuration are needed in the development of supercapacitors. Indeed, a very large effective surface is required to get a high capacity and this can be provided by highly porous layers. 


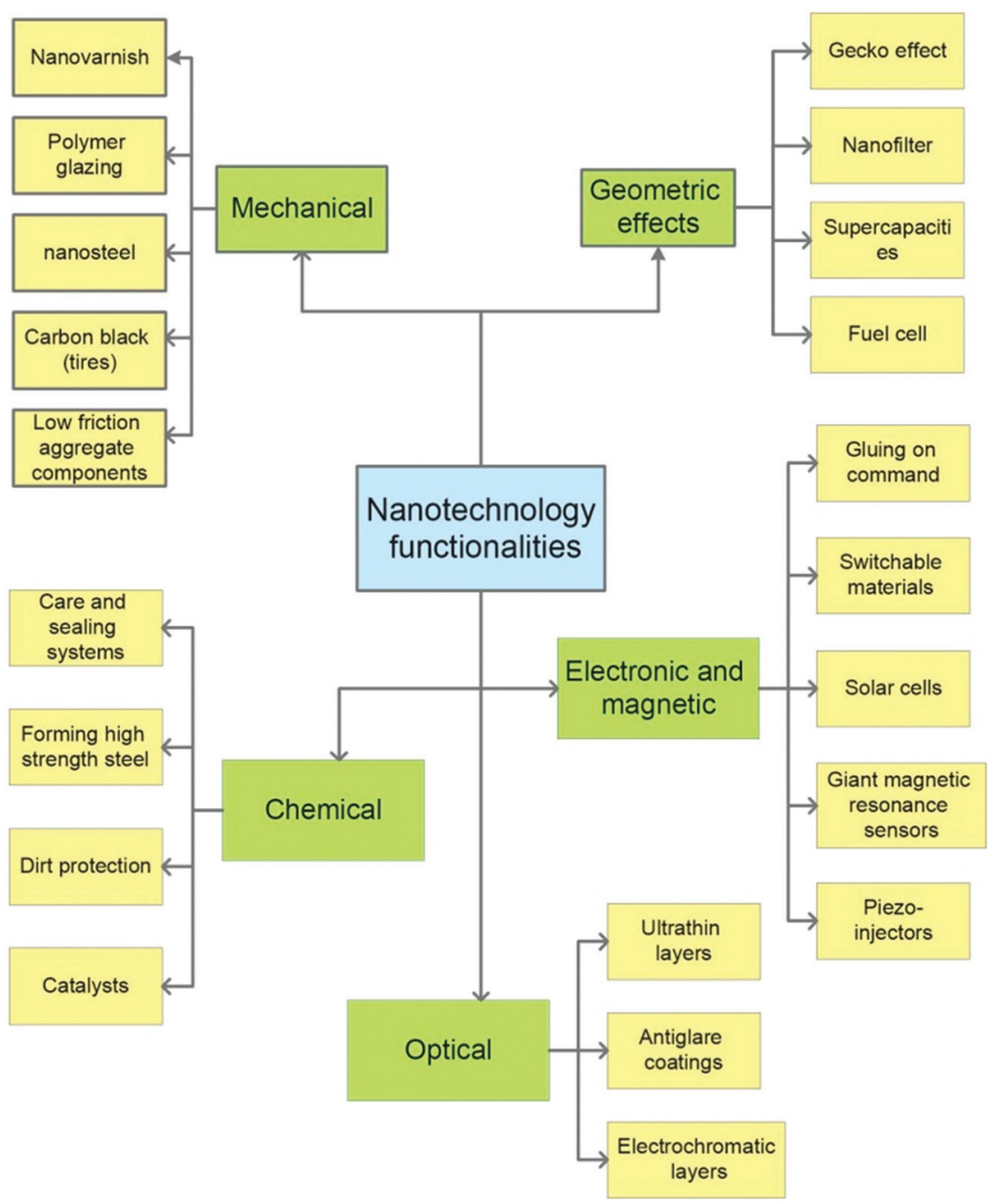

Figure 136. Applications of nanotechnology in the automotive sector. Built from the report "Nanotechnologies in automobiles". Innovation potential in Hessen for the automotive industry and its subcontractors, http://www.hessen-nanotech.de.

Nanotechnology will play an even more important role in the next generation batteries or supercapacitors and in fuel cells of the future vehicles to be fueled by hydrogen, etc.

\section{Summary}

Each second, about 13 cars are manufactured in the world. This corresponded, in 2011, to the manufacture of 76 million cars. There are now more 
than one billion vehicles in the world and the automotive industry represents a large part of the world economy.

Figure 136 summarizes areas of nanotechnology either already existing or under investigation for use in automobiles. 